\title{
Tuning plasticity of in-situ dendrite metallic glass composites via the dendrite-volume-fraction-dependent shear banding
}

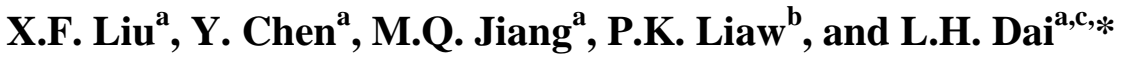 \\ ${ }^{a}$ State Key Laboratory of Nonlinear Mechanics, Institute of Mechanics, Chinese \\ Academy of Sciences, Beijing 100190, China \\ ${ }^{b}$ Department of Materials Science and Engineering, The University of Tennessee, \\ Knoxville, TN 37996, USA \\ ${ }^{\mathrm{c}}$ State Key Laboratory of Explosion Science and Technology, Beijing Institute of \\ Technology, Beijing 100081, China
}

\begin{abstract}
This work performs a systematic investigation of identifying how the volume fraction of the in-situ dendrites affects the plasticity of metallic glass composites. The quasi-static uniaxial compressions show that the global plastic strain does not follows a linear rule-of-mixture with the dendrite volume faction, instead, a slow-fast-slow enhancement behaviour is observed with increasing dendrite volume fraction. It is demonstrated that the nucleation and propagation of shear bands in these composites are dependent on the dendrite volume fraction. When the dendrite volume fraction exceeds a critical value, multiple shear bands emerge in a spherical plastic zone around a dendrite. It is further proposed that the percolation of these spherical plastic zones contributes to the fast increase in the plastic strain of the glass composites. Our findings offer important implications for the microstructural optimization of the metallic glass composites with desirable mechanical properties.
\end{abstract}


Keywords: Metallic glass Composites; Shear band; Volume fraction; Plasticity; Percolation

\section{Introduction}

Bulk metallic glasses (BMGs) have attracted extensive interests due to their remarkable properties, such as high strength, extraordinary hardness, and excellent wear resistance [1-5]. However, at room temperature, BMGs usually undergo the plastic flow localization into nanoscale shear bands [6-9]. Uncontrolled shear banding can incur catastrophic failure with very limit ductility $[6,10]$, impeding wide applications of BMGs as structural materials. An effective way to solve this bottle-neck problem is to develop BMG composites by in-situ introducing crystalline second phases [11-21]. It is expected that these crystalline phases facilitate shear band nucleation at their interfaces with the glass matrix, and meanwhile act as obstacles to shear band propagation into cracks due to their relatively high shear-band toughness $[22,23]$. However, the presence of crystalline phases tends to decrease the global strength of composites as compared to pure matrix glasses. Therefore, sustained efforts have been made to optimize the strength and plasticity of composites by modifying the sizes, spacing, shape, distribution and volume fraction of crystalline phases [13, 24-31]. Among these, the volume fraction is a structural parameter to effectively tune the composite's properties. It was found that, the plastic deformation exhibits a nonlinear enhancement as the volume fraction of crystalline phases increases above a critical value $[13,27,32]$. Lee et 
al. [13] and Liu et al. [32] have ascribed this phenomenon to percolation of shear banding, corresponding to a specific configuration of crystalline phases' distribution. However, the picture of how shear banding evolves in composites with increasing volume fraction of crystalline phase remains unclear up to now, and the relationship between the percolation point of global plasticity and the spatial configuration of crystalline phases also deserves further investigation. In this study, Zr-based BMGCs containing different volume fractions of dendrites were synthesized. We then investigate how the dendrite volume fraction affects shear banding behaviours and the plasticity, using interrupted compression technique and microscopic image analysis. A percolation transition, i.e., a brittle-to-plastic transition, was found with increasing dendrite volume fraction. The shear banding behaviours were revealed below and above the percolation threshold.

\section{Materials and methods}

According to the pseudo-ternary phase diagram with apexes of $\mathrm{Zr}, \mathrm{Ti}+\mathrm{Nb}$, and $\mathrm{Be}_{9} \mathrm{Cu}_{5} \mathrm{Ni}_{4}$ [33], as shown in Fig. 1, six alloys were designed along the line of $\left(\mathrm{Zr}_{75} \mathrm{Ti}_{15} \mathrm{Nb}_{10}\right)_{100-\mathrm{y}}\left(\mathrm{Be}_{50} \mathrm{Cu}_{27.5} \mathrm{Ni}_{22.5}\right)_{\mathrm{y}}$ with $\mathrm{y}=20,26,27,29,30$ and 32. It was shown that $\mathrm{Be}, \mathrm{Cu}$, and $\mathrm{Ni}$ are distributed preferentially in the glass matrix, and the composites with different volume fractions of the dendrites can be obtained via changing the value, ' $y$ ' [33]. The master ingots of the six composites were prepared by melting the mixture of high-purity $\mathrm{Zr}, \mathrm{Ti}, \mathrm{Nb}, \mathrm{Cu}, \mathrm{Ni}$, and Be elements (>99.9 wt. \%) under a Ti-gettered argon atmosphere. Afterwards, the ingots were crushed into pieces and remelted by 
induction under a high pure argon atmosphere, and subsequently, cast into a copper mould with a 10-mm-diameter cavity. The phases of the samples were examined by X-ray diffraction (XRD) in a Philips PW1050 diffractometer using $\mathrm{Cu} \mathrm{Ka}$ radiation. Rectangular bar specimens $(7 \times 3.5 \times 3.5 \mathrm{~mm})$ for mechanical tests were taken from the central area of the cylinders by electric spark machining. Compression tests were conducted using a MTS 810 mechanical testing machine under a strain rate of $5 \times 10^{-4}$ $\mathrm{s}^{-1}$ at room temperature. The microstructures of the as-cast samples, the lateral surfaces and fracture surfaces of the deformed samples were investigated by scanning electron microscopy (SEM) with an energy dispersive X-ray spectrometer (EDS). Before the SEM observations, the lateral surfaces of the unreformed samples were mechanically polished by using the $1-\mu \mathrm{m}$-diamond paste. The microstructures of the composites before and after compressions were observed by transmission electron microscopy (TEM) in a JEM-2100F instrument. The TEM samples were prepared by mechanically grinding, followed by ion beam thinning using a Gatan duo-ion mill equipped with a liquid-nitrogen-cooled stage.

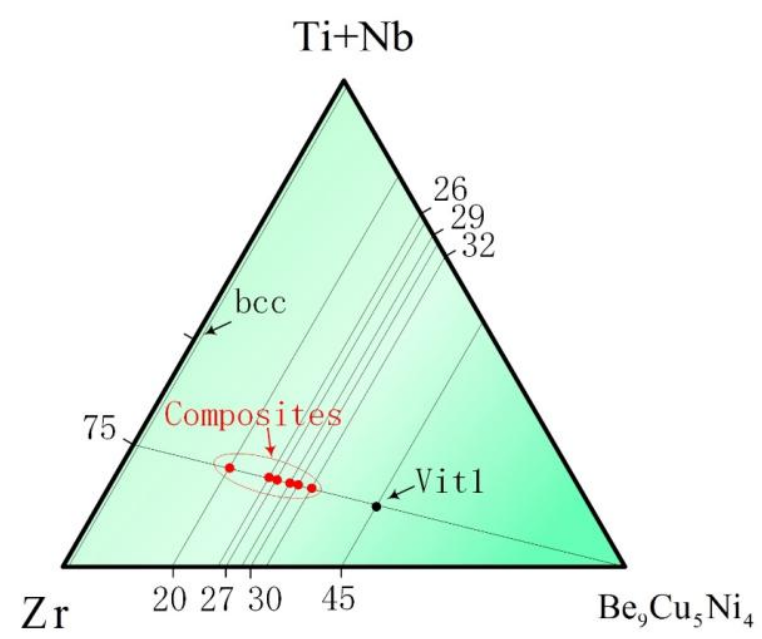


Figure 1. Pseudo-ternary phase diagram with apexes of $\mathrm{Zr}$, $(\mathrm{Ti}+\mathrm{Nb})$, and $\mathrm{Be}_{9} \mathrm{Cu}_{5} \mathrm{Ni}_{4}$. Compositions of the fabricated composites (red spots) and a typical monolithic metallic glass Vit 1 (black spot) are marked.

\section{Results}

\subsection{Material characterization}

Figure 2a shows the XRD patterns of the as-cast 10-mm-diameter rods of the six alloys. It can be seen that intense crystalline peaks, marked as different crystalline planes of the body-centred cubic (b.c.c) solid solution, are superimposed on a broad diffuse scattering maximum of the amorphous matrix. This demonstrates that all the six alloys have a two-phase microstructure. It is noted that by decreasing the portion of $\mathrm{Be}_{50} \mathrm{Cu}_{27.5} \mathrm{Ni}_{22.5}$ in the composition, the intense crystalline peaks increase remarkably, but the broad diffraction peak becomes less obvious. Figure $2 b$ shows a TEM image of one typical alloy with the composition $\left(\mathrm{Zr}_{75} \mathrm{Ti}_{15} \mathrm{Nb}_{10}\right)_{100-\mathrm{y}}\left(\mathrm{Be}_{50} \mathrm{Cu}_{27.5} \mathrm{Ni}_{22.5}\right)_{\mathrm{y}}(\mathrm{y}=29)$, with light and dark areas corresponding to the dendrites and the glass matrix, respectively. The high-resolution TEM (HRTEM) image in Fig. 2c, taken from the rectangle in Fig. 2b, exhibits the clear and tight interface between the two phases. The selected area electron diffraction patterns (SAED) in the insets of Fig. 2c show the typical glass matrix and the b.c.c crystalline dendrites, respectively, confirming the dual-phase structure of the composites. 

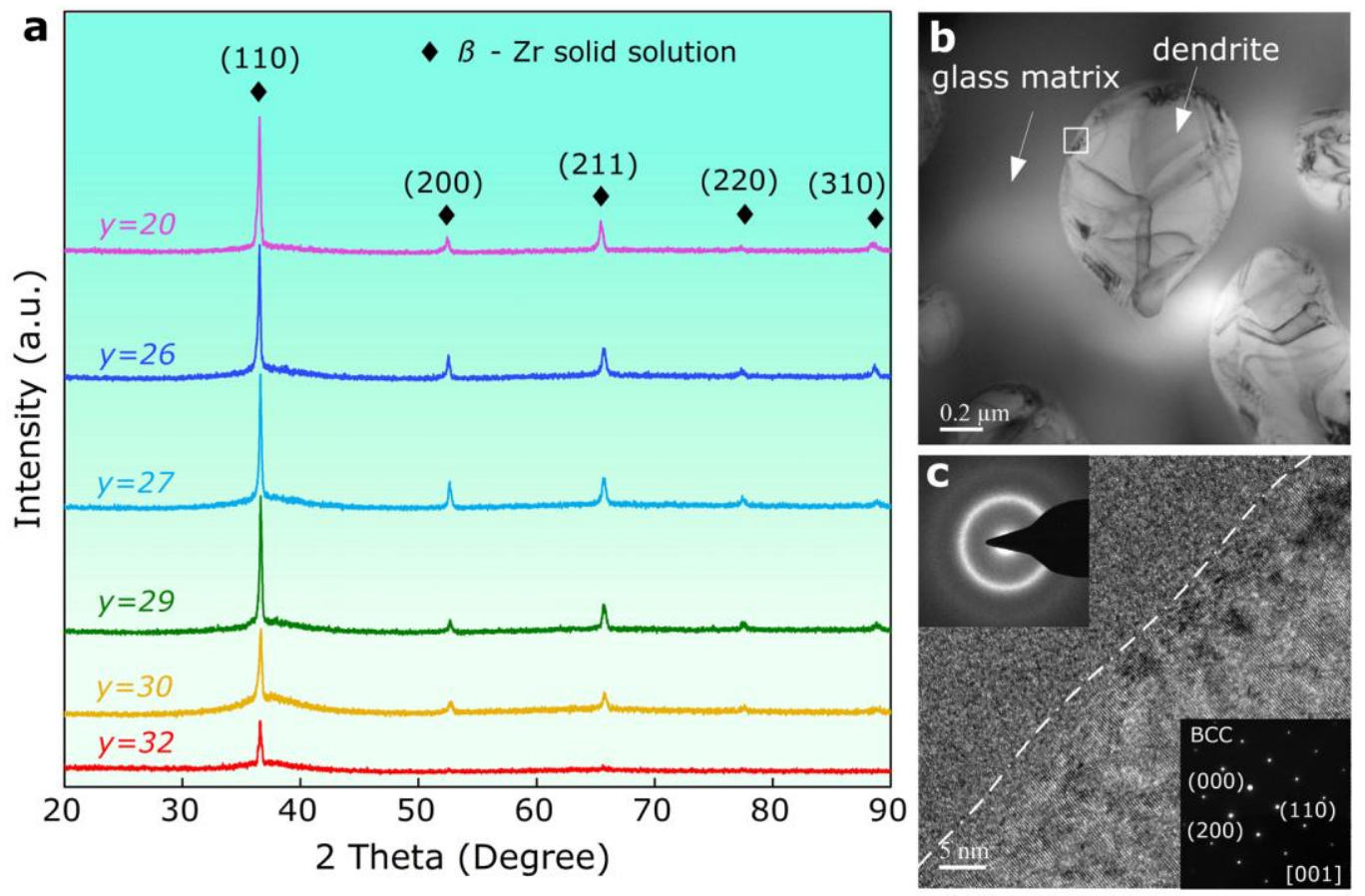

Figure 2. (a) XRD patterns of the as-cast $10 \mathrm{~mm}$ diameter cylinders of the composites $\left(\mathrm{Zr}_{75} \mathrm{Ti}_{15} \mathrm{Nb}_{10}\right)_{100-\mathrm{y}}\left(\mathrm{Be}_{50} \mathrm{Cu}_{27.5} \mathrm{Ni}_{22.5}\right)_{\mathrm{y}}$ with different value, y. The TEM image (b) of the composite with $y=29$ at low magnification and its HRTEM image (c) of the interface taken from the rectangle in (b). Insets in (c) on the upper left corner and the bottom right corner are the SAED patterns corresponding to the glass matrix and the dendrites, respectively.

Figure 3 presents the characteristic microstructures of the six composites observed by the SEM backscattered electron mode, exhibiting the tendency of the dendrite volume fraction $f_{d}$ increasing with the decreased value of $\mathrm{y}$, which is consistent with the XRD results. By analysis of greyscale values of SEM images, $f_{d}$ is determined for each alloy. It has been revealed that crystals grouped closely are actually have the same orientation and form a single dendrite [31]. For the alloy with an $8 \%$ dendrite volume fraction, the individual dendrite size is about $20 \mu \mathrm{m}$, and the dendrites are sparsely 
distributed among the glass matrix. As y decreases to 20, corresponding to the value of $f_{d} 55 \%$, the dendrites are nearly touched each other, while the dendrite size remains the same. The microstructure characters of the composites, such as the volume fraction, dendrite size, dendrite arm diameter as well as the composition of the dendrites, are summarized in Table 1.

Table 1

Microstructures of BMGC (Zr75Ti15Nb10) ${ }_{100-\mathrm{y}}(\mathrm{Be} 50 \mathrm{Cu} 27.5 \mathrm{Ni} 22.5)_{\mathrm{y}}(\mathrm{y}=20-32)$

\begin{tabular}{|c|c|c|c|c|}
\hline Alloy (Label ${ }^{1}$ ) & $\begin{array}{c}\text { Dendritic volume } \\
\text { Fraction }(\%)\end{array}$ & $\begin{array}{l}\text { Dendritic } \\
\operatorname{size}^{2}(\mu \mathrm{m})\end{array}$ & $\begin{array}{l}\text { Dendritic arm } \\
\text { diameter }(\mu \mathrm{m})\end{array}$ & $\begin{array}{c}\text { Composition of } \\
\text { dendrites }\end{array}$ \\
\hline $\mathrm{y}=32,(\mathrm{~A} 08)$ & $8 \pm 4$ & $15 \pm 5$ & $0.5 \sim 2$ & $\mathrm{Zr}_{61.4} \mathrm{Nb}_{13.4} \mathrm{Ti}_{12} \mathrm{Ni}_{5.6} \mathrm{Cu}_{7.6}$ \\
\hline $\mathrm{y}=30,(\mathrm{~A} 21)$ & $21 \pm 3$ & $22 \pm 5$ & $1 \sim 3$ & $\mathrm{Zr}_{68} \mathrm{Nb}_{16} \mathrm{Ti}_{12} \mathrm{Ni}_{1.5} \mathrm{Cu}_{2.5}$ \\
\hline $\mathrm{y}=28,(\mathrm{~A} 25)$ & $25 \pm 3$ & $16 \pm 3$ & $1.5 \sim 3$ & $\mathrm{Zr}_{66.8} \mathrm{Nb}_{19} \mathrm{Ti}_{11} \mathrm{Ni}_{1.1} \mathrm{Cu}_{2.1}$ \\
\hline $\mathrm{y}=27,(\mathrm{~A} 32)$ & $32 \pm 3$ & $20 \pm 3$ & $1.5 \sim 2.5$ & $\mathrm{Zr}_{65} \mathrm{Nb}_{19} \mathrm{Ti}_{12} \mathrm{Ni}_{1.5} \mathrm{Cu}_{2.5}$ \\
\hline$y=26,(A 38)$ & $38 \pm 2$ & $25 \pm 5$ & $2 \sim 4$ & $\mathrm{Zr}_{68.3} \mathrm{Nb}_{17} \mathrm{Ti}_{11} \mathrm{Ni}_{1.4} \mathrm{Cu}_{2.5}$ \\
\hline $\mathrm{y}=20,(\mathrm{~A} 55)$ & $55 \pm 3$ & $21 \pm 4$ & $2 \sim 4$ & $\mathrm{Zr}_{67} \mathrm{Nb}_{15.8} \mathrm{Ti}_{12.1} \mathrm{Ni}_{2} \mathrm{Cu}_{2.5}$ \\
\hline \multicolumn{5}{|c|}{$\begin{array}{l}\text { 1. Alloys with different volume fractions of dendrites are referred to as the labels in the } \\
\text { brackets for brevity, respectively. } \\
\text { 2. The dendritic size and the dendritic arm diameter were obtained by analysing the } \\
\text { backscattered SEM images. }\end{array}$} \\
\hline
\end{tabular}



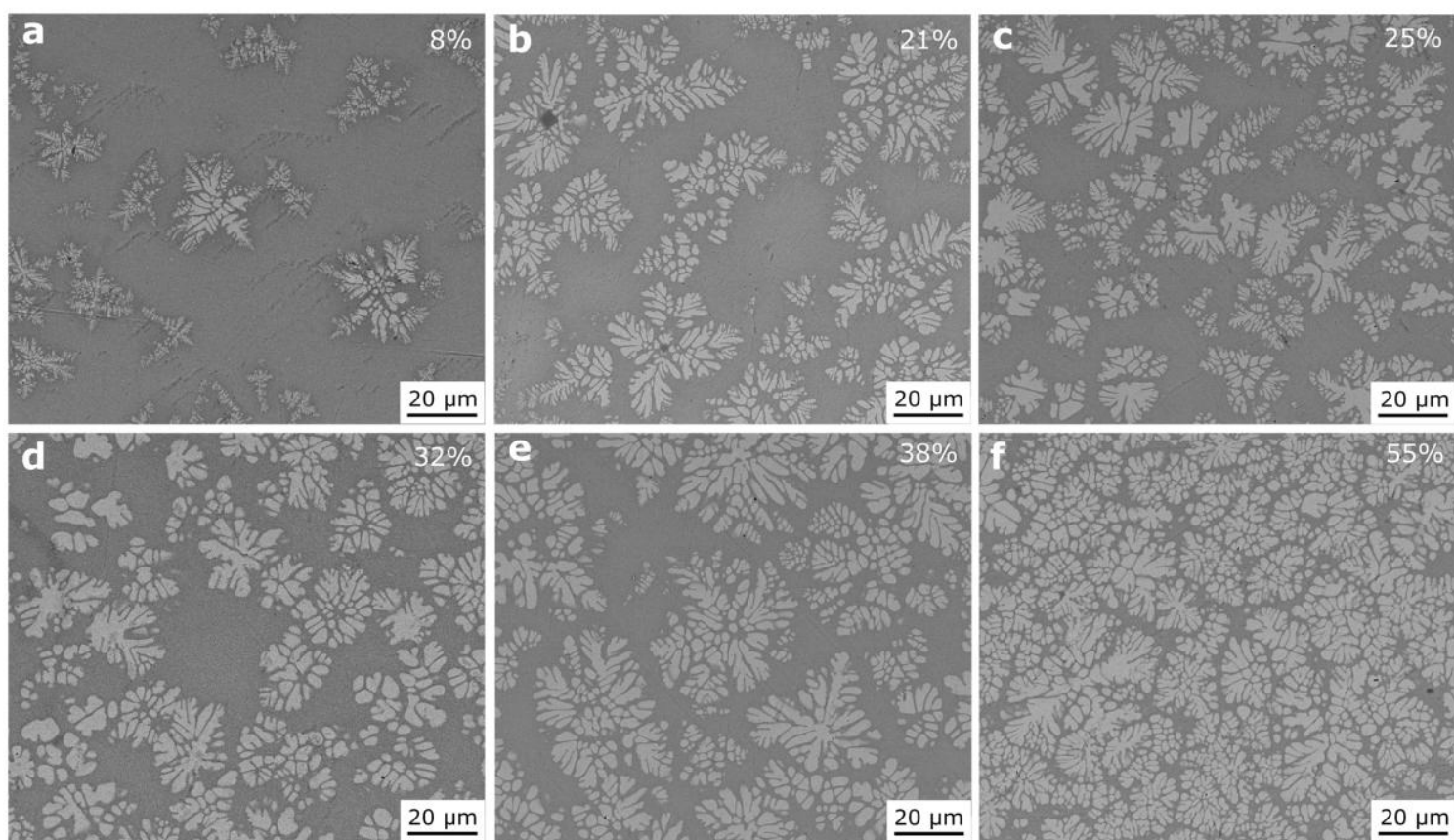

Figure 3. The dendrites appears bright, where the glass matrix appears dark. The compositions are $\left(\mathrm{Zr}_{75} \mathrm{Ti}_{15} \mathrm{Nb}_{10}\right)_{100-\mathrm{y}}\left(\mathrm{Be}_{50} \mathrm{Cu}_{27.5} \mathrm{Ni}_{22.5}\right)_{\mathrm{y}}$ with (a) $\mathrm{y}=32, f_{\mathrm{d}}=8 \%$, (b) $\mathrm{y}=$ $30, f_{\mathrm{d}}=21 \%$, (c) y $=29, f_{\mathrm{d}}=25 \%$, (d) $\mathrm{y}=27, f_{\mathrm{d}}=32 \%$, (e) $\mathrm{y}=26, f_{\mathrm{d}}=38 \%$, and (f) $\mathrm{y}=$ $20, f_{\mathrm{d}}=55 \%$.

\subsection{Uniaxial Compression}

Figure $4 \mathrm{a}$ shows the uniaxial compressive stress-strain curves. The A8 alloy (referred to as the composite containing $8 \%$ of dendrites, see Table I) fractures catastrophically at the strength of $1,780 \pm 35 \mathrm{MPa}$ without detectable plasticity, similar to its glass matrix [33]. With the increase in $f_{d}$, the composites show the decreased yield strength $Y_{c}$ but increased plasticity $\varepsilon_{c}$, as manifested by the fractured samples in the inset of Fig. 4a. To exhibit the effect of $f_{d}$ on the mechanical properties, Figure $4 \mathrm{~b}$ plots the variation of $Y_{c}$ and $\varepsilon_{c}$ with $f_{d}$. The absorbed energy $E$, the area beneath the stress-strain curve that was used as a definition of toughness [34, 35], was also added in Figure 4b. One can see that $Y_{c}$ decreases linearly with the increase in $f_{d}$, 
which can be reasonably described by the rule of mixtures $[13,32]$ :

$$
Y_{c}=Y_{d} f_{d}+Y_{m} f_{m}
$$

where $Y$ and $f$ are the yield strength and the volume fraction of the constituent phases, and the subscripts $c, m$ and $d$ refer to the composite, glass matrix and dendrite, respectively. The linear fitting of the experimental data gives the values of $Y_{d}=660 \mathrm{MPa}, Y_{m}=1900 \mathrm{MPa}$, in agreement with the previous results $[33,36]$. As opposed to the yield strengths, a nonlinear enhancement of the plasticity was observed $[13,32]$, showing the percolation transition, i.e., the onset of macroscopic plasticity [37, 38 , around the critical volume fraction $21 \%$. And the plastic strains versus the volume fraction exhibits an S-shape curve that can be separated into three regions: Region I with $f_{d} \leq 21 \%$, Region II with $21 \%<f_{d}<32 \%$, and Region III with $f_{d} \geq 32 \%$, indicating different shear banding behaviours in the composites with different dendrite volume fraction, which will be discussed below. The absorbed energy $E$ exhibits the similar trend to that of the plastic strain. In the range of the volume fractions of dendrites from $21 \%$ to $32 \%$, the toughness increases significantly; whereas out of this range, the toughness remains almost unchanged. 

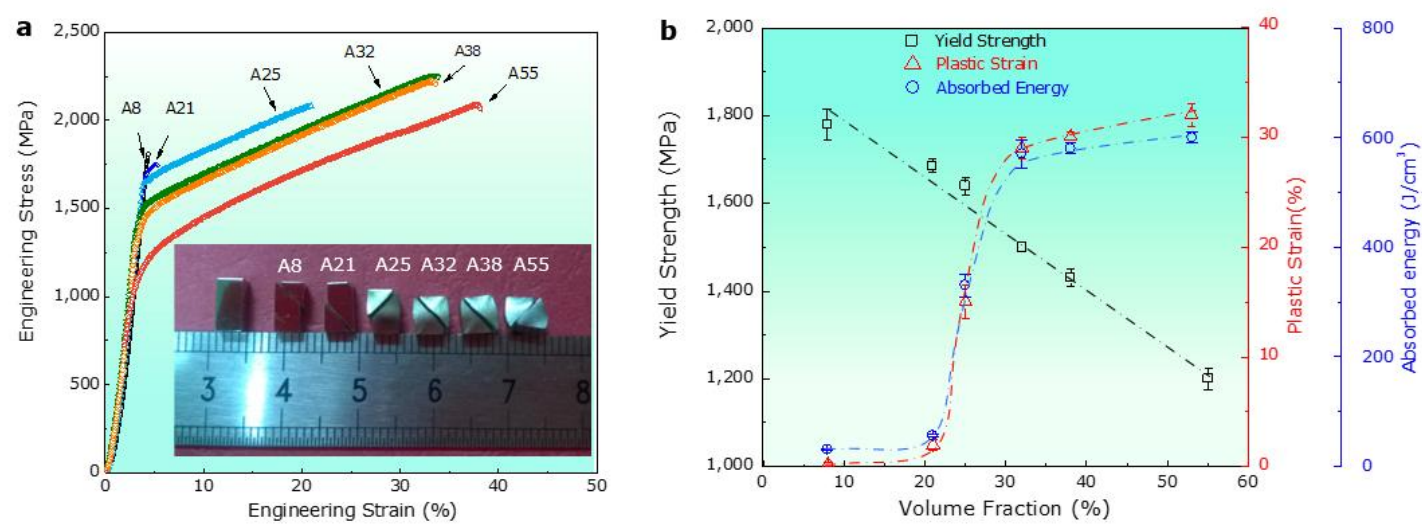

Figure 4. (a) Engineering stress-strain curves for the six composites. Macroscopically fractured samples shown in the inset. (b) Relations of yield strengths, plastic strains and absorbed energy with the dendritic volume fraction. The $\mathrm{S}$-shape line is added to guide the eyes.

Figure 5 presents the morphologies of the side and fracture surfaces of the fractured composites. For the A8 alloy and A21 alloy, only a few shear bands were seen on the lateral surfaces (Figs. 5a, b). This agrees well with their stress-strain curves without obvious plasticity (Fig. 4a). The main shear band penetrated through the whole samples, and developed into a crack rapidly, resulting in the catastrophic failure. However, as $f_{d}$ increased beyond $25 \%$, multiple shear bands prevailed in the glass matrix, and most of the shear bands were confined to comparatively isolated regions, forming shear band arrays (Figs. 5c-f), due to the rapid propagation of shear bands hindered by the dendrites. These shear bands propagated stably and collectively sustained further applied strains, and delayed localized shear-off through the entire sample, which resulted in the improved plasticity of the composites. Upon large deformation, some shear bands penetrated the dendrites and developed into microcracks (Figs. 5c-f). It is likely that the growth and coalescence of these microcracks gave rise to the final rupture of the 
composites $[39,40]$. Figures $5 \mathrm{~g}$ and $\mathrm{h}$ show the fracture surfaces of the A8 and A32 alloys respectively, which are characteristic of macroscopically brittle and plastic failure of the composites. The A8 alloy exhibits the microscale cells and river-like patterns, typical in many monolithic BMGs [10]. The fracture surface of the A32 alloy shows two distinct features, one being relatively smooth with some striations and the other displaying droplet-like patterns. The morphologies suggest that a larger value of $f_{d}$ makes the propagation of shear bands more stable in the composites, thus sustaining more global plasticity. The SEM images clearly demonstrated different shear banding behaviours after the percolation transition.
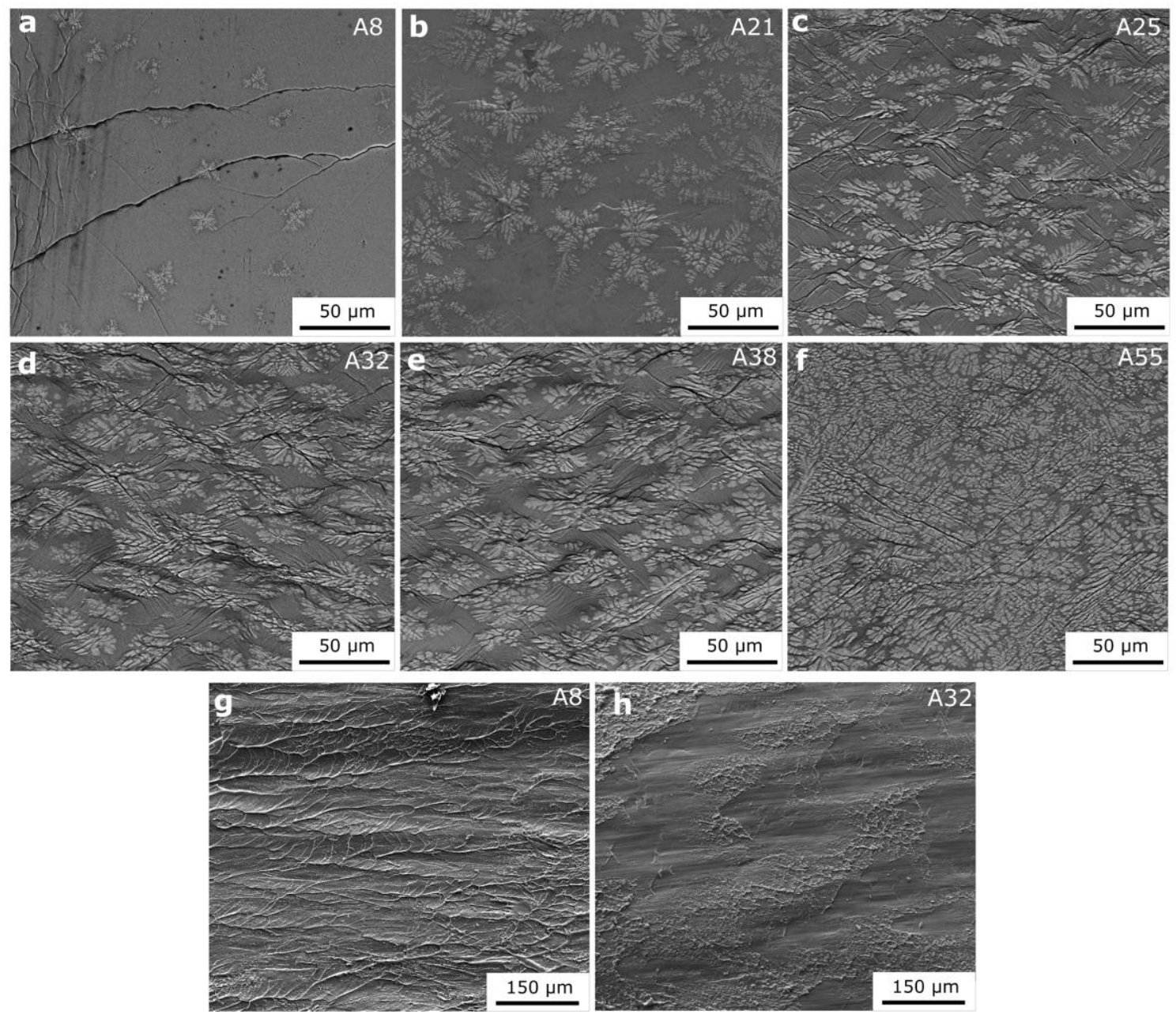
Figure 5. (a)-(f) Backscattering SEM images of the side surfaces of the fractured samples with different dendrite volume fractions (Marks in the upper right corner of each image referred from Table I). (g), (h) SEM images of fracture surfaces of the A8 alloy and the A32 alloy, respectively.

\subsection{Interrupted compression}

To understand the shear band evolution during deforming the composites, interrupted compression tests were conducted on the A25 composite. The macroscopic stress-strain curve and typical microstructural evolution are shown in Fig. 6. The first loading stopped at the engineering stress of 1,500 MPa. From the enlarged stress-strain curve in the left inset, a strain burst can be seen, indicating the shear band activation. The corresponding SEM observation in Fig. $6 \mathrm{~b}$ shows that shear bands are initiated at the glass matrix/dendrite interfaces, denoted by arrows. Due to the different elastic constants of the two phases, there exists a stress concentration at the interfaces where shear bands initiate although the average stress in the glass matrix is lower than its yield stress. Thus, the increased applied stress is required for the already-existing shear bands to satisfy the critical condition for propagation, giving rise to the apparent work-hardening behaviour [41]. When the stress increased to 1,650 MPa, the onset of the steady plastic flow occurred, and distinct serrations with stress drops of about 10 MPa were observed (the right inset in Fig. 6a). Upon further loading, the already-existing shear bands propagated into the glass matrix further, and some were arrested by other shear bands in another direction or stopped at the interfaces, as indicated by the arrows in Fig. 6c. Moreover, new shear bands were generated. It is 
reasonable to speculate that the more dendrites precipitate, the more shear bands will initiate by more interactions between shear bands and dendrites. Comparing Fig. 6c with d, the density of shear bands did not continuously grow rapidly when the samples are strained to some extent $(\sim 11 \%)$. As a consequence, continued shearing on the pre-exiting shear planes started to play the dominant role in bearing further deformation. Accompanied by such a procedure, excess free volume that was generated within the shear bands led to void nucleation due to their coalescence [42]. The voids then induced the formation of a local microcrack. As the shear cracks propagated and encountered the interfaces, some of them penetrated the dendrites because of the intense stress concentration at the crack tip, and the network formed by the dendrites to block shear bands or cracks was then broken down gradually (Fig 6d). Finally, an unstable crack formed by the coalescence of wildly distributed microcracks rapidly transected the samples and led to the fracture of materials.

Figure 6e shows the TEM image of the deformed A25 alloy sample just before fracture. The selected area electron diffraction (SAED) pattern in the inset reveals that the dendrites retained their crystalline structure upon loading without phase transformation. The dark lines or the dark regions in the dendrite are identified as dense dislocation walls (DDWs) [43], formed by dense tangled dislocations to harmonize the applied strains, which divide the individual dendrite into several subdivisions. Additionally, sharp corners with steps of $200 \sim 300 \mathrm{~nm}$ were observed, indicative of the shearing of the dendrite resulting from the impediment of the propagating shear bands, 
in accordance with the severe distortion of the dendrites (Figs. 6b-d). Compared with the undeformed sample (Fig. 2c), the interface between the two phases became fuzzy because of the intense interactions of shear bands and dendrites (Fig. 6f). Moreover, nanocrystals with the size of several nanometres were found in the glass matrix, denoted by the ellipse and the corresponding fast-Fourier transformed (FFT) pattern shown in the upper inset of Fig. 6f. It should be noted that such nanocrystals are induced during deformation because no nanocrystals were found in the undeformed samples (Fig. 2c). An early study revealed that atomic diffusion in the metallic glass is greatly improved by the applied stress, suggesting that the higher stresses near the interfaces facilitate the local relaxation [44]. The inverse fast-Fourier transformed (IFFT) pattern for the area, marked by the rectangle, is displayed in the lower inset of Fig. 6f. Lattice distortion and pile-ups of edge dislocations were observed near the interface, giving the evidence of the dendrite deformation behaviours to accommodate the strain. 

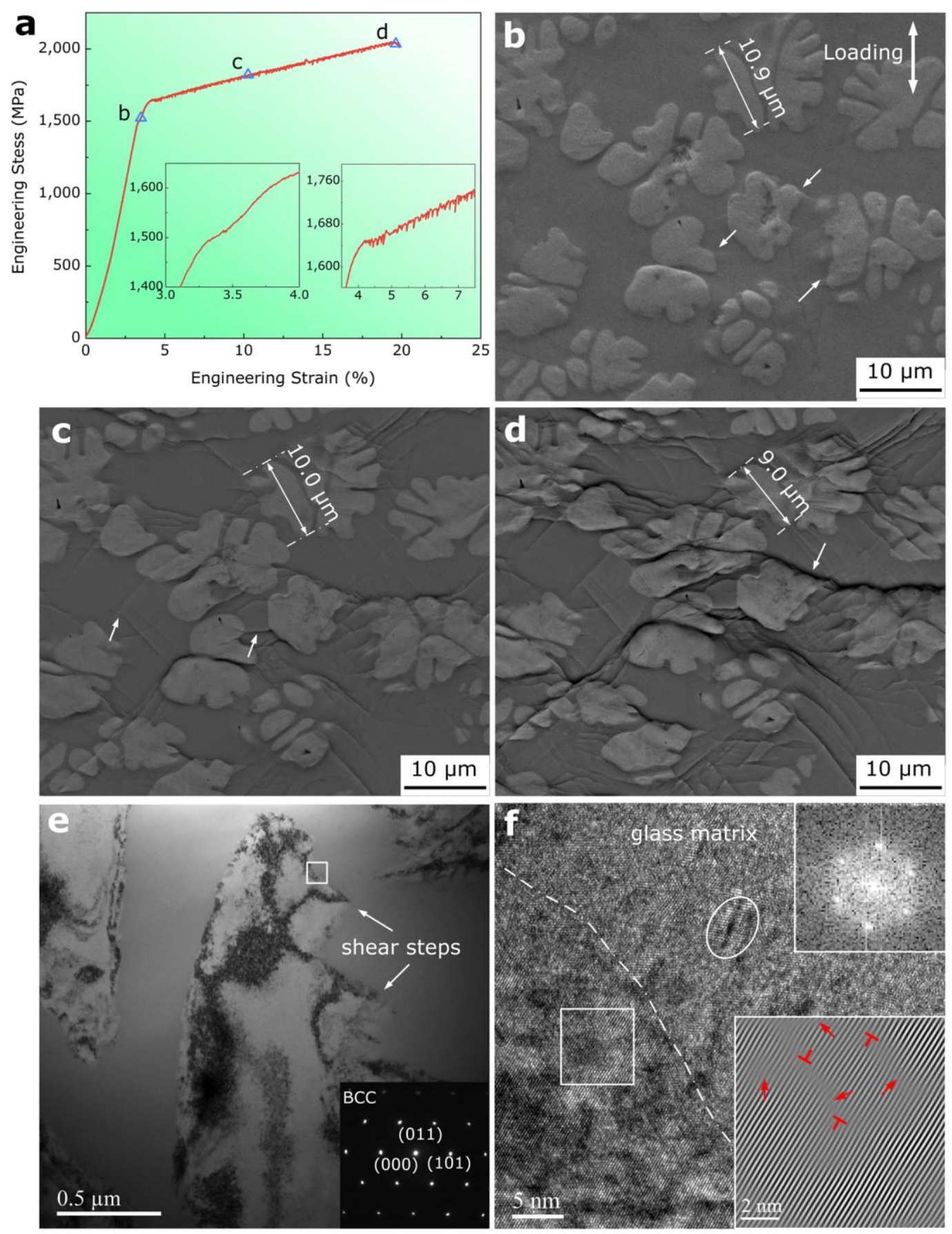

Figure 6. (a) The compressive stress-strain curve of the composites containing a $25 \%$ dendrite volume fraction (A25 alloy). The left and right insets show the enlarged portion of stress-strain curve at the onset of shear banding and onset of steady flow, respectively. (b), (c), (d), the lateral surfaces of the sample deformed to different deformation stages in (a), respectively. (e) The TEM image of deformed A25 alloy sample just before fracture. (Inset) SAED pattern of the dendrite. (f) HRTEM image near the interface in (e), marked by the rectangle; the upper inset is the corresponding FFT patterns of the ellipse, the lower inset is the corresponding IFFT image of the rectangle in (f), lattice distortion and dislocations are marked, respectively. 


\section{Discussion}

The experiment results clearly show that the mechanical properties of the present BMGCs are greatly affected by the volume fraction of dendrites, and there exists a percolation transition around the critical value $21 \%$, due to different shear banding behaviours across this percolation threshold.

It is well known that the plastic strain of metallic glass at room temperature results from the shear displacement of two part separated by the shear band [45]. With the shear band propagating, the free volume generated will lower the bonding strength $[22,46]$. When the shear displacement reaches a critical value, the catastrophic final fracture always proceeds along the shear-softening band [47, 48]. If it were possible to limit the rapid propagation of individual shear bands and generate multiple shear bands, the plastic strain would be distributed over many bands. Then the onset of fracture might be delayed and significant macroscopic plastic strains could be realized $[1,6]$. For the present BMGCs, dendrites are introduced to realize the purpose. According to previous work, shear band toughness $K_{c}$ was introduced in crystalline alloys [49] and metallic glass[22], which was utilized to measure the critical energy $\Gamma_{c}$ in a shear band, by $K_{c}=\sqrt{2 G \Gamma_{c}}$ with the shear modulus $G$. If the energy released exceeds its $\Gamma_{c}$, the shear banding will become a runway shear crack. As revealed by Jiang and Dai [22], the $\Gamma_{c}$ in metallic glasses is determined by the balance between the free volume diffusion and the momentum dissipation. That is very different from the $\Gamma_{c}$ in crystalline phases which is determined by the balance between the thermal diffusion and the momentum 
dissipation. For the quasi-static cases, the shear band toughness $K_{c}$ of the dendrite phase is estimated to be of the order of $10^{2} \mathrm{MPam}^{1 / 2}$. But the glassy matrix only has the $K_{c}$ of $\sim 10^{0}-10^{1} \mathrm{MPam}^{1 / 2}[23]$. The higher shear band toughness of the crystalline phase make it possible that the dendrites can act as ductile obstacles for the propagation of the shear band.

However, only beyond the percolation threshold, corresponding to the matrix spacing or the length of the ligament $T$ lower than a critical value, can the composites demonstrate plasticity. With the increase in dendrite volume fraction $f_{d}, T$ decreases, then the mean free path for shear band to propagate will be shortened. Moreover, shear band nucleation is easier to take place than propagation, as Chen et al. [50] found in monolithic metallic glass under bending that the samples of smaller sizes give rise to shear band multiplication and thus obtain larger plasticity. In this regard, we suggest that the brittle-to-plastic transition can be formulated as the percolation (i.e. connectivity) of thin ligaments, where multiple shear banding is favoured over crack opening.

Inspired by Margolina and Wu's interpretation on the brittle-tough transition in nylon/rubber blends [38], we assume that there exists an outer sphere regarded as a spherical plastic zone (SPZ), inside which shear band multiplication is prone to occur, centred at a dendrite and including a concentric annular shell of a critical matrix spacing $T_{c} / 2$. Namely, the diameter of the SPZ is $S=T_{c}+D$, where $D$ is the diameter of the crystalline particle, as shown in Fig. 7. When the volume fraction of the SPZs, $f_{s}$, 
reaches a critical value, where thin ligaments accompanied with multiple shear banding pervades the entire specimen and no cracks form in the early stage of loading, the brittle-to-plastic transition takes place. Since the volume fraction of the SPZs, $f_{s} \sim S^{3}$, the volume fraction of the dendrites, $f_{d} \sim D^{3}$, we obtain

$$
f_{s}=f_{d}\left(S^{3} / D^{3}\right)=f_{d}\left(\mathrm{~T}_{\mathrm{c}}+D\right)^{3} / D^{3}
$$

To avoid catastrophic failure requires that the SPZ should be distributed among the whole sample. Then, shear bands emitted from different sites and in different orientations will not develop into fast propagating cracks, because they can be effectively blocked by the dendrites or shear-band intersections. Thus, the critical value of $f_{s}$ is 0.65 , a typical value of the dense random packing of hard spheres [51]. Under the assumption that dendrites are of the same size, substitute $f_{s}, f_{d}$ in Equation (2) with $0.65,0.21$, then we have $T_{c}=0.46 D$. Noting that reinforcement particles act both as nucleation sites as well as obstacles to shear band propagation, the spatial configuration of the crystalline phases at the percolation threshold will be dependent on details of the glass composition and structure, as well as, e.g., the shape and average length scale of the reinforcement phases $[13,27,32]$. 

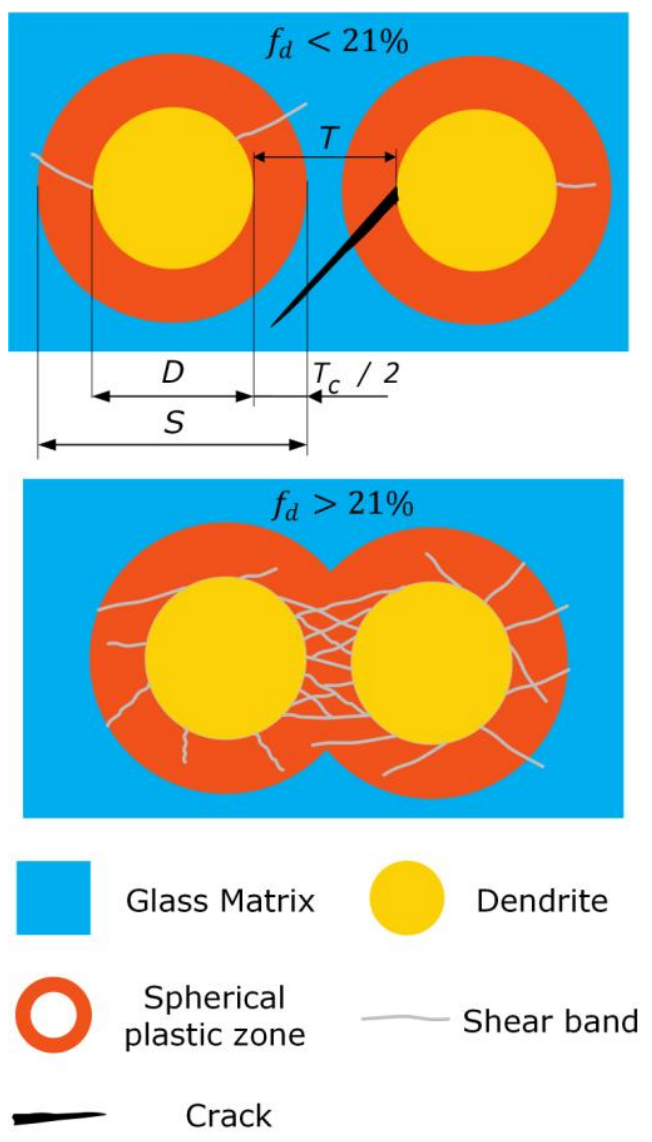

Figure 7. Illustration of interactive neighbouring dendrites in composites with low dendritic volume fractions $\left(f_{d}<21 \%\right)$, and high volume fractions $\left(f_{d}>21 \%\right)$. Shear bands prevail between two neighbouring dendrites as the surrounded spherical plastic zones percolate, corresponding to the composites with high dendrite volume fraction that have improved plasticity.

To figure out the shear banding behaviours of the specific spatial configuration of the dendrites more, we examine the stress distribution around a dendrite and the effect of the spacing between dendrites on the stress field. For the ease of the mathematical treatment, it is assumed that the dendrites are spherical particles with the same diameter in all composites [36]. When a soft phase reinforced composite is applied by a uniaxial 
stress, the hoop stress that appears on the equatorial plane of the particle, in the same direction as the applied stress, is much larger than other stress components. Usually, the stress-induced shear bands are observed to initiate at the equatorial plane of a soft crystal, as shown in Fig. 6b or in other report [39]. Considering this phenomenon, the distributions of the hoop stress around the particle on the equatorial plane are calculated only. According to Goodier's solutions [52], the local stress at any point around the particle is obtained by superposing the additional stress on the applied stress. Thus, the stress distributions are calculated:

$$
\begin{aligned}
& \sigma=\sigma_{a d}+\sigma_{\infty}=\sigma_{\infty}\left[-2(\alpha+5 \beta)\left(\frac{a}{r}\right)^{3}+36 \beta\left(\frac{a}{r}\right)^{5}\right]+\sigma_{\infty} \\
& \alpha=\frac{1}{8} \frac{\mu_{1}-\mu_{2}}{\left(7-5 v_{1}\right) \mu_{1}+\left(8-10 v_{1}\right) \mu_{2}} \frac{2\left(1-2 v_{2}\right)\left(6-5 v_{1}\right) \mu_{1}+\left(3+19 v_{2}-20 v_{1} v_{2}\right) \mu_{2}}{2\left(1-2 v_{2}\right) \mu_{1}+\left(1+v_{2}\right) \mu_{2}} \\
& +\frac{1}{4} \frac{\left[\left(1-v_{1}\right)\left(\frac{1+v_{2}}{1+v_{1}}\right)-v_{2}\right] \mu_{2}-\left(1-2 v_{2}\right) \mu_{1}}{2\left(1-2 v_{2}\right) \mu_{1}+\left(1+v_{2}\right) \mu_{2}} \\
& \beta=\frac{1}{8} \frac{\mu_{1}-\mu_{2}}{\left(7-5 v_{1}\right) \mu_{1}+\left(8-10 v_{1}\right) \mu_{2}}
\end{aligned}
$$

where $a$ is the radius of the particle, $r$ is the distance from the centre of the particle, $\mu$ is the shear modulus, $v$ is Poisson's ratio, and the subscripts, 1 and 2, refer to the matrix and particle, respectively. The parameters used to calculate the stresses are as follows: $\mu_{1}=33 \mathrm{GPa}, \mu_{2}=23 \mathrm{GPa}, v_{1}=0.369$, and $v_{2}=0.4$ [33]. As shown in Fig. 8, the stress concentrations $\sigma_{33} / \sigma_{\infty}$ are quite localized and fall off rapidly within the distance from the particle about one radius away (the red curve marked by squares). As $f_{d}$ increases, $T$ becomes smaller, and the stress field around a particle will be 
influenced by its neighbouring particle-induced stress field. By assuming a simple additivity of the two stress fields, the stress concentration at point $\mathrm{P}$, varied with the distance between the centres of two neighbouring particles, $l$, is calculated (the blue curve marked by triangles). As $l$ is larger than $3 a$, the stress concentration factor will be almost the same as that of one single particle. Comparing the rapid change in stress in the region where $l$ is smaller than $3 \mathrm{a}(T<0.5 D)$ with the percolation occurrence at $T_{c}=0.46 D$ as aforementioned, we may infer that the enhanced stress fields promote the formation of the multiple shear bands when $f_{d}$ is beyond the threshold, which is also suggested by FEM simulations in Liu et al.'s work [32].

According to the above analysis, the picture of how shear banding evolves before and after the percolation of shear banding, and its effects on the plastic deformation of the composites become clear. Below the percolation threshold, a fast propagating crack proceeds along the shear plane once the primary shear band travels a certain distance, while most of the sample deform elastically. As a result of the crack's rapid transecting 


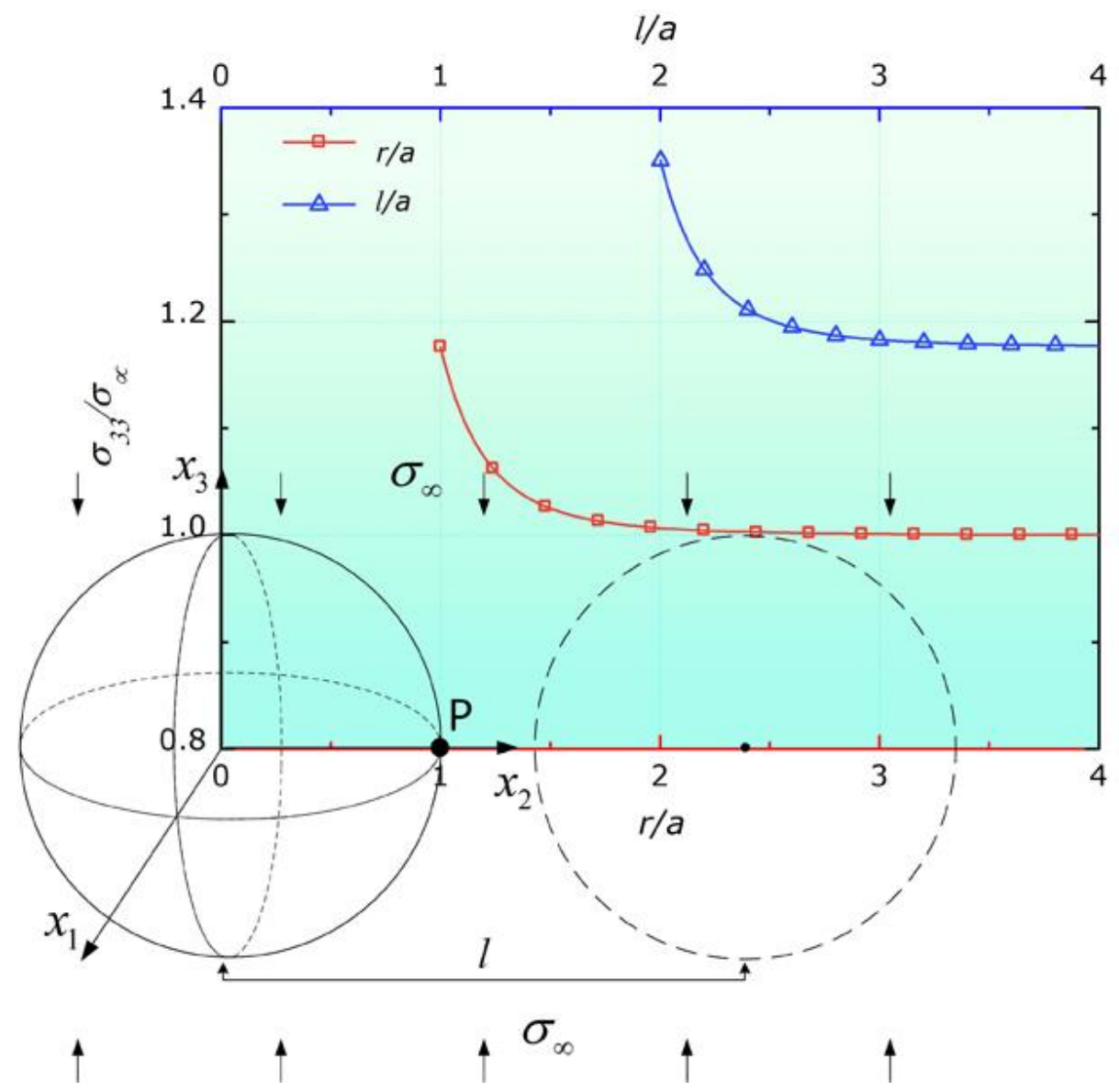

Figure 8. The stress concentration factor in the glass matrix along the $x_{2}$ axis is given, marked by the squares. The curve marked by the triangles indicates the stress concentration factor varied with the distance, $l$, between two neighbouring particles at point $\mathrm{P}$.

the specimen, only few shear bands are formed and the composites fail catastrophically. Thus the composites still deform in a brittle manner, similar to its monolithic glass matrix (see Fig. 5a and 5b). With $f_{d}$ increasing beyond the percolation threshold, the SPZs pervade the whole specimen, giving rise to the predominance of stabilized shear banding rather than individual shear bands evolving into cracks in the early stage. 
Therefore, more shear bands will be initiated by more interactions between shear bands and dendrites, as well as shear band intersections to sustain further loading (see Fig. 6). The enhanced plasticity of the composites tends to improve rapidly with increasing $f_{d}$.

However, the plasticity levels off to an almost plateau value when $f_{d}$ reaches $32 \%$. Once a shear band initiates and subsequently propagates, the flow stress decreases, due to the free-volume softening within the shear band, which will lead to unloading of its neighbouring medium $[22,50]$. This stress relaxation then produces a shield against the nucleation of new shear bands. Thus, the density of shear bands will not continuously grow rapidly, which can be seen in Figs. 5c-f. Furthermore, according to the evolution of shear bands in Fig. 6, the increased plastic strains result mainly from the slip along the pre-exiting shear bands after a certain strain $(\sim 10 \%)$. The shear bands then penetrate the dendrites or converge to form a microcrack along the interfaces, which results in the prevalence of microcracks among the samples (Figs. 5c-f). The easy connection of these microcracks incurs the final fracture of the composites, and the rapid improvement of plasticity ceases although $f_{d}$ still increases.

\section{Conclusions}

This work investigated the effects of the dendrite volume fraction on the quasi-static compression behaviour of the BMGCs with the compositions of $\left(\mathrm{Zr}_{75} \mathrm{Ti}_{15} \mathrm{Nb}_{10}\right)_{100-\mathrm{y}}\left(\mathrm{Be}_{50} \mathrm{Cu}_{27.5} \mathrm{Ni}_{22.5}\right)_{\mathrm{y}}(\mathrm{y}=20-32)$. The yield strength generally obeys a linear rule-of-mixture, while the plastic strain exhibits a nonlinear relation with the volume fraction. A rapid increase in the plastic strain is observed in the volume fraction 
ranging from $21 \%$ to $32 \%$. The improved plasticity is attributed to the homogeneously distributed dendrites, which act as the shear-band-initiation sites and the block of shear band propagation. At the critical dendrite volume fraction of $21 \%$, the stress field around a dendritic particle starts to be enhanced by its neighbouring dendritic particles to form an SPZ, where multiple shear bands can be generated. Then, the percolation of the SPZs contributes to the onset of the rapidly increased plasticity. However, owing to the saturated density of shear bands and the cracking behaviours after large deformation, the fast increase ends in spite of the growth of the dendrite volume fraction. As a result, the metallic glass composites exhibit the slow-fast-slow enhancement of plastic strains with increasing the dendrite volume fraction. Finally, our study of the dendrite-volume-fraction-dependent shear banding can guide the design and preparation of metallic glass composites. By changing the volume fraction of dendrites, the strength and the ductility of the composites can be tailored to satisfy the requirement of their service condition, which will help to extend their potential engineering applications.

\section{Acknowledgments}

Financial support is from the National Key Basic Research Program of China (Grant No. 2012CB937500), the NSFC (Grants Nos. 11472287, 11132011, 11572324, 11372315, 11522221) and the CAS/SAFEA International Partnership Program for Creative Research Teams. P.K. Liaw would like to acknowledge the Department of Energy (DOE), Office of Fossil Energy, National Energy Technology Laboratory (DE-FE-0008855, DE-FE-0024054, and DE-FE-0011194), with Mr. V. Cedro, Mr. R. 
Dunst, and Dr. J. Mullen as program managers. P.K. Liaw very much appreciates the support of the U.S. Army Research Office project (W911 NF-13-10438) with the program manager, Dr. D. M. Stepp. P.K. Liaw thanks the support from the National Science Foundation (CMMI-1100080) with the program director, Dr. C. Cooper.

\section{References}

[1] C. Schuh, T. Hufnagel, U. Ramamurty, Mechanical behavior of amorphous alloys, Acta Mater. 55(12) (2007) 4067-4109.

[2] W.H. Wang, C. Dong, C.H. Shek, Bulk metallic glasses, Mater. Sci. Eng. R. 44(2-3) (2004) 45-89.

[3] M. Chen, Mechanical behavior of metallic glasses: Microscopic understanding of strength and ductility, Annu. Rev. Mater. Res. 38 (2008) 445-469.

[4] M. Ashby, A. Greer, Metallic glasses as structural materials, Scripta Mater. 54(3) (2006) 321-326.

[5] J. Qiao, H. Jia, P.K. Liaw, Metallic glass matrix composites, Mater. Sci. Eng. R 100 (2016) 1-69.

[6] A.L. Greer, Y.Q. Cheng, E. Ma, Shear bands in metallic glasses, Mater. Sci. Eng. R 74(4) (2013) 71-132.

[7] L.H. Dai, Y.L. Bai, Basic mechanical behaviors and mechanics of shear banding in BMGs, Int. J. Impact Eng 35(8) (2008) 704-716.

[8] M.Q. Jiang, L.H. Dai, On the origin of shear banding instability in metallic glasses, J. Mech. Phys. Solids 57(8) (2009) 1267-1292.

[9] B. Yang, M.L. Morrison, P.K. Liaw, R.A. Buchanan, G. Wang, C.T. Liu, M. Denda, Dynamic evolution of nanoscale shear bands in a bulk-metallic glass, Appl. Phys. Lett. 86(14) (2005) 141904.

[10] M.Q. Jiang, Z. Ling, J.X. Meng, L.H. Dai, Energy dissipation in fracture of bulk metallic glasses via inherent competition between local softening and quasi-cleavage, Philos. Mag. 88(3) (2008) 407-426.

[11] C. Hays, C. Kim, W. Johnson, Microstructure controlled shear band pattern formation and enhanced plasticity of bulk metallic glasses containing in situ formed ductile phase dendrite dispersions, Phys. Rev. Lett. 84(13) (2000) 2901-2904.

[12] U. Kuhn, J. Eckert, N. Mattern, L. Schultz, ZrNbCuNiAl bulk metallic glass matrix composites containing dendritic bcc phase precipitates, Appl. Phys. Lett. 80(14) (2002) 2478-2480.

[13] M. Lee, Y. Li, C. Schuh, Effect of a controlled volume fraction of dendritic phases on tensile and compressive ductility in La-based metallic glass matrix composites, Acta Mater. 52(14) (2004) 4121-4131.

[14] Z. Bian, H. Kato, C. Qin, W. Zhang, A. Inoue, Cu-Hf-Ti-Ag-Ta bulk metallic 
glass composites and their properties, Acta Mater. 53(7) (2005) 2037-2048.

[15] Y.C. Kim, E. Fleury, J.-C. Lee, D.H. Kim, Origin of the simultaneous improvement of strength and plasticity in Ti-based bulk metallic glass matrix composites, J. Mater. Res. 20(09) (2005) 2474-2479.

[16] K. Wang, T. Fujita, D. Pan, T.G. Nieh, A. Inoue, D.H. Kim, M.W. Chen, Interface structure and properties of a brass-reinforced Ni59Zr20Ti16Si2Sn3 bulk metallic glass composite, Acta Mater. 56(13) (2008) 3077-3087.

[17] D.C. Hofmann, J.Y. Suh, A. Wiest, G. Duan, M.L. Lind, M.D. Demetriou, W.L. Johnson, Designing metallic glass matrix composites with high toughness and tensile ductility, Nature 451(7182) (2008) 1085-9.

[18] S. Pauly, S. Gorantla, G. Wang, U. Kühn, J. Eckert, Transformation-mediated ductility in CuZr-based bulk metallic glasses, Nat. Mater. 9(6) (2010) 473-477.

[19] Y. Wu, Y. Xiao, G. Chen, C.T. Liu, Z. Lu, Bulk Metallic Glass Composites with Transformation - Mediated Work - Hardening and Ductility, Adv. Mater. 22(25) (2010) 2770-2773.

[20] Z. Chen, J. Gao, Y. Wu, H. Wang, X. Liu, Z. Lu, Designing novel bulk metallic glass composites with a high aluminum content, Sci. Rep. 3 (2013).

[21] C. Jeon, C.P. Kim, H.S. Kim, S. Lee, Quasi-static and dynamic compressive properties of Ti-based amorphous alloys modified from conventional Ti-6Al-4V alloy, Mater. Sci. Eng. A 607 (2014) 197-205.

[22] M.Q. Jiang, L.H. Dai, Shear-band toughness of bulk metallic glasses, Acta Mater. 59(11) (2011) 4525-4537.

[23] J. Chen, M. Jiang, Y. Chen, L. Dai, Strain rate dependent shear banding behavior of a Zr-based bulk metallic glass composite, Mater. Sci. Eng. A 576 (2013) 134-139.

[24] G.Y. Sun, G. Chen, G.L. Chen, Comparison of microstructures and properties of Zr-based bulk metallic glass composites with dendritic and spherical bcc phase precipitates, Intermetallics 15(5-6) (2007) 632-634.

[25] R. Narayan, P. Singh, D. Hofmann, N. Hutchinson, K. Flores, U. Ramamurty, On the microstructure-tensile property correlations in bulk metallic glass matrix composites with crystalline dendrites, Acta Mater. 60(13) (2012) 5089-5100.

[26] Y. Wu, H. Wang, H. Wu, Z. Zhang, X. Hui, G. Chen, D. Ma, X. Wang, Z. Lu, Formation of $\mathrm{Cu}-\mathrm{Zr}-\mathrm{Al}$ bulk metallic glass composites with improved tensile properties, Acta Mater. 59(8) (2011) 2928-2936.

[27] Z. Liu, R. Li, G. Liu, W. Su, H. Wang, Y. Li, M. Shi, X. Luo, G. Wu, T. Zhang, Microstructural tailoring and improvement of mechanical properties in CuZr-based bulk metallic glass composites, Acta Mater. 60(6-7) (2012) 3128-3139.

[28] X.L. Fu, Y. Li, C.A. Schuh, Temperature, strain rate and reinforcement volume fraction dependence of plastic deformation in metallic glass matrix composites, Acta Mater. 55(9) (2007) 3059-3071.

[29] D.C. Hofmann, J.Y. Suh, A. Wiest, M.L. Lind, M.D. Demetriou, W.L. Johnson, Development of tough, low-density titanium-based bulk metallic glass matrix composites with tensile ductility, Proc. Natl. Acad. Sci. USA 105(51) (2008) 20136-40. [30] J.W. Qiao, J.T. Zhang, F. Jiang, Y. Zhang, P.K. Liaw, Y. Ren, G.L. Chen, 
Development of plastic Ti-based bulk-metallic-glass-matrix composites by controlling the microstructures, Mater. Sci. Eng. A 527(29-30) (2010) 7752-7756.

[31] C. Jeon, M. Kang, C.P. Kim, H.S. Kim, S. Lee, Quasi-static and dynamic compressive deformation behaviors in Zr-based amorphous alloys containing ductile dendrites, Mater. Sci. Eng. A 579 (2013) 77-85.

[32] Z. Liu, G. Liu, R. Qu, Z. Zhang, S. Wu, T. Zhang, Microstructural percolation assisted breakthrough of trade-off between strength and ductility in CuZr-based metallic glass composites, Sci. Rep. 4 (2014).

[33] F. Szuecst, C. Kim, W. Johnson, Mechanical properties of Zr56. 2Ti13. 8Nb5. 0Cu6. 9Ni5. 6Be12. 5 ductile phase reinforced bulk metallic glass composite, Acta Mater. 49(9) (2001) 1507-1513.

[34] M.S. Khorrami, M.A. Mostafaei, H. Pouraliakbar, A.H. Kokabi, Study on microstructure and mechanical characteristics of low-carbon steel and ferritic stainless steel joints, Mater. Sci. Eng. A 608 (2014) 35-45.

[35] M.R. Jandaghi, H. Pouraliakbar, Study on the effect of post-annealing on the microstructural evolutions and mechanical properties of rolled CGPed Aluminium-Manganese-Silicon alloy, Mater. Sci. Eng. A DOI: 10.1016/j.msea.2016.10.05.

[36] B. Clausen, S. Lee, E. Üstündag, C. Kim, D. Brown, M. Bourke, Compressive deformation of in situ formed bulk metallic glass composites, Scripta Mater. 54(3) (2006) 343-347.

[37] R. Zallen, The physics of amorphous solids, Wiley, New York, 1983.

[38] A. Margolina, S. Wu, Percolation model for brittle-tough transition in nylon/rubber blends, Polymer 29(12) (1988) 2170-2173.

[39] G. Chen, J. Cheng, C. Liu, Large-sized Zr-based bulk-metallic-glass composite with enhanced tensile properties, Intermetallics 28 (2012) 25-33.

[40] Y. Huang, J. Khong, T. Connolley, J. Mi, Understanding the deformation mechanism of individual phases of a ZrTi-based bulk metallic glass matrix composite using in situ diffraction and imaging methods, Appl. Phys. Lett. 104(3) (2014) 031912.

[41] R. Ott, F. Sansoz, J. Molinari, J. Almer, K. Ramesh, T. Hufnagel, Micromechanics of deformation of metallic-glass-matrix composites from in situ synchrotron strain measurements and finite element modeling, Acta Mater. 53(7) (2005) 1883-1893.

[42] W.J. Wright, T. Hufnagel, W. Nix, Free volume coalescence and void formation in shear bands in metallic glass, J. Appl. Phys. 93(3) (2003) 1432-1437.

[43] J. Qiao, A. Sun, E. Huang, Y. Zhang, P. Liaw, C. Chuang, Tensile deformation micromechanisms for bulk metallic glass matrix composites: From work-hardening to softening, Acta Mater. 59(10) (2011) 4126-4137.

[44] S. Lee, M. Huh, S. Chae, J. Lee, Mechanism of the deformation-induced nanocrystallization in a $\mathrm{Cu}$-based bulk amorphous alloy under uniaxial compression, Scripta Mater. 54(8) (2006) 1439-1444.

[45] C.A. Pampillo, Flow and fracture in amorphous alloys, Journal of Materials Science 10(7) (1975) 1194-1227.

[46] X. Lei, Y. Wei, Z. Hu, W.-H. Wang, Strength softening at shear bands in metallic 
glasses, Philos. Mag. Lett. 93(4) (2013) 221-230.

[47] R.D. Conner, W.L. Johnson, N.E. Paton, W.D. Nix, Shear bands and cracking of metallic glass plates in bending, J. Appl. Phys. 94(2) (2003) 904-911.

[48] G. Ravichandran, A. Molinari, Analysis of shear banding in metallic glasses under bending, Acta Mater. 53(15) (2005) 4087-4095.

[49] D. Grady, Properties of an adiabatic shear-band process zone, J. Mech. Phys. Solids 40(6) (1992) 1197-1215.

[50] Y. Chen, M. Jiang, L. Dai, Collective evolution dynamics of multiple shear bands in bulk metallic glasses, Int. J. Plast. 50 (2013) 18-36.

[51] S. Safran, I. Webman, G.S. Grest, Percolation in interacting colloids, Phys. Rev. A 32(1) (1985) 506.

[52] J. Goodier, Concentration of stress around spherical and cylindrical inclusions and flaws, J. Appl. Mech 55(7) (1933) 39-44. 